\title{
Theoretische Perspektiven auf Programmplanung in der Erwachsenenbildung
}

\section{Eine Systematisierung von Programmplanungsmodellen für Forschung und Praxis}

\author{
Aiga von Hippel (D)
}

Eingegangen: 28. Mai 2017 / Angenommen: 4. August 2017 / Online publiziert: 14. August 2017

(C) Der/die Autor(en) 2017. Dieser Artikel ist eine Open-Access-Publikation.

Zusammenfassung Weiterbildungsprogramme zeigen gesellschaftliche Auslegungen von Bildung und sind das Ergebnis pädagogisch-professionellen Handelns. Die Forschung zu Weiterbildungsprogrammen und den dahinterliegenden Planungsprozessen ist Bildungsforschung, die Bildungsbegriff(e) phänomenologisch rekonstruiert und die eine Wissensbasis für gesellschaftlich auszuhandelnde Entscheidungen legt. Programmplanungsmodelle stellen dabei Teilperspektiven auf Programmplanung dar. Ein Ziel des Beitrags ist es, einen Überblick über Programmplanungsmodelle zu geben. Eine Systematik der Modelle ist wichtig, da sie die unterschiedlichen Anschlüsse und Fragestellungen für Forschung und Praxis aufzeigt und zu einer begründeten Modellauswahl in Forschung und Praxis beitragen kann. Der Beitrag legt eine neu erarbeitete Systematik zur Differenzierung von Programmplanungsmodellen und ihre Verknüpfung mit Fragestellungen vor.

Schlüsselwörter Programm · Programmplanung · Programmforschung · Programmplanungsmodelle

\section{Theoretical perspectives on program planning in adult education}

A systematization of program-planing models for research and practice

Abstract Programs of further education reflect societal interpretations of education and are the result of professional pedagogical action. Research on programs of

Prof. Dr. A. von Hippel $(\bowtie)$

Kultur-, Sozial- und Bildungswissenschaftliche Fakultät, Institut für Erziehungswissenschaften, Abteilung Erwachsenenbildung/Weiterbildung, Humboldt Universität zu Berlin,

Unter den Linden 6, 10099 Berlin, Deutschland

E-Mail: aiga.von.hippel@hu-berlin.de

Prof. Dr. A. von Hippel

Geschwister-Scholl-Straße 7, Raum 407, 10117 Berlin, Deutschland 
further education and on the underlying planning processes is educational research that reconstructs educational concepts in a phenomenological manner and that provides a knowledge base for decisions that need to be negotiated by society. In this context, program-planning models constitute partial perspectives on program planning. One goal of the article is to give an overview of program-planning models. A classification of these models is important because it reveals the diverse links and questions to be dealt with in both research and practice and it contributes to a well-founded model selection in research and practice. The article's main focus is to present a newly developed classification of program-planning models and its linkages to research questions.

Keywords Program · Program planning $\cdot$ Program research $\cdot$ Program-planning models

\section{Einleitung}

Der Erwachsenenbildungsbereich ist dadurch gekennzeichnet, dass in weiten Teilen keine festen, staatlich vorgegebenen Curricula und keine staatlich für alle reglementierte Teilnahmepflicht bestehen (insbesondere die allgemeine Erwachsenenbildung beruht auf freiwilliger Teilnahme) (vgl. Schrader und Ioannidou 2011). Daraus folgt, dass auf makro-/mesodidaktischer Ebene kontinuierlich Bildungsangebote und -programme für unterschiedliche Interessen geplant werden müssen (vgl. von Hippel 2011). Programmplanung hat damit eine grundlegende Bedeutung für die Erwachsenenbildung. Das Programm - als Ergebnis professionell-pädagogischen Planungshandelns - ist konstitutiv für die Erwachsenenbildung (vgl. Gieseke 2000). In ihm spiegeln sich gesellschaftliche Auslegungen von (Erwachsenen-)Bildung mit kontextspezifischen (institutionellen, organisatorischen, professionellen) Re-Spezifikationen.

Die Programmforschung in der Erwachsenenbildung untersucht mit unterschiedlichen Zielstellungen Programmplanungshandeln und das Ergebnis des Handelns, das Programm. Programme werden typischerweise mit qualitativen und/oder quantitativen Programmanalysen untersucht (für einen systematischen Forschungsüberblick über Programmanalysen, teilweise nach Kategorien differenziert vgl. Gieseke 2015; Käpplinger 2008; Robak 2012; Schrader und Ioannidou 2011; Nolda 2015; DIE 2017). Das Planungshandeln selbst wird in überwiegend qualitativen Untersuchungsdesigns mit Interviews, Beobachtungen und Dokumentenanalysen untersucht (vgl. Gieseke 2000).

Es liegen nur wenige umfangreiche empirische Studien zum Programmplanungshandeln vor. Als eine umfangreiche empirische Studie, die das Programmplanungshandeln fokussiert, ist die Studie von Gieseke (2000) zu nennen. Es gibt wenige Studien, die Programmanalysen mit Analysen des Programmplanungshandelns verknüpfen, teilweise auch mit Analysen der Teilnehmendenseite (vgl. Gieseke 2000; Heuer und Robak 2000; Robak et al. 2015; von Hippel und Röbel 2016). Als weitere Studie, die das Planungshandeln untersuchte, ist beispielsweise Dollhausen (2008) zu nennen. Rezipientenstudien gibt es insgesamt nur wenige, d. h. Studien, die da- 
nach fragen, wie Adressaten die Programme wahrnehmen (vgl. Schrader und Ioannidou 2011). Als Rezipientenstudien sind insbesondere das Projekt ImZiel zu nennen (vgl. Tippelt et al. 2008; von Hippel 2008) sowie die Studie von Gnahs (1995) zum Informationsgehalt von Ankündigungen. Während es Systematiken der Programmforschung (insbesondere zu Programmanalysen) gibt, gibt es bislang wenige Systematisierungen zu den Modellen zur Programmplanung und ihrer Verknüpfung mit Forschung(sfragen).

Der vorliegende Beitrag gibt einen Überblick über Programmplanungsmodelle in der Erwachsenenbildung. Ziel des Beitrags - neben dem Überblick - ist es, Modelle zum Programmplanungshandeln nach Kategorien zu systematisieren und mit typischen empirischen Fragestellungen und -zielen zu verbinden - was bislang ein Desiderat darstellte (bislang wurde hauptsächlich in interaktive und linear-zyklische Modelle unterschieden, vgl. von Hippel und Käpplinger 2017; McLean 2000). Eine Systematik zeigt die unterschiedlichen Anschlüsse und Fragestellungen für Programmforschung und Praxis auf und trägt zu einer begründeten Modellauswahl bei.

\section{Theoretische Perspektiven auf Programmplanung}

Im Folgenden erfolgt die Beschreibung theoretischer Perspektiven auf Programmplanung mit Schwerpunkt aus dem deutschsprachigen Raum. Während in England ursprünglich eher auf implizite Curriculumtheorien Bezug genommen wurde, war die Diskussion in Nordamerika stärker explizit und fokussiert auf programme planning (mittlerweile trifft dies auch auf England zu) (vgl. Jarvis 2006, S. $244 \mathrm{ff}$.). Aus diesem Grund sollen hier zusätzlich nordamerikanische Perspektiven einbezogen werden (vgl. zum internationalen Modellvergleich von Hippel und Käpplinger 2017). International vergleichend lässt sich sagen, dass es eine Vielzahl nordamerikanischer Programmplanungsmodelle gibt, wobei leitend für Forschung hauptsächlich das Modell von Cervero und Wilson (1994) ist. Programmarchive und systematische Programmanalysen wie sie kennzeichnend sind für den deutschsprachigen Raum, scheint es international gesehen nicht zu geben ${ }^{1}$ (vgl. auch Herre 2015). D. h. der internationale Blick nach Nordamerika lohnt sich für einige Modelle, ist aber nicht instruktiv in Bezug auf Programmanalysen, die die Entwicklung und den IstStand des gesellschaftlichen Angebots für lebenslanges Lernen abbilden können.

\subsection{Theorien und Modelle und ihr Vergleich}

Theorien beschreiben durchschauend und verstehend Phänomene in ihren Strukturen und erklären sie, d. h. Theorien enthalten beschreibende (deskriptive) und erklärende (kausale) Aussagen (manche Theorien enthalten mehr beschreibende, manche mehr erklärende Aussagen). Sie sind sowohl Folge wissenschaftlicher Erkenntnis-

\footnotetext{
${ }^{1}$ Dies zeigten Gespräche mit KollegInnen und Recherchen (über ESREA; Adult Education Quarterly; AAACE; internationale Konferenz zur Programmplanung „Cultures of Program Planning in Adult Education“vgl. Käpplinger et al. 2017).
} 
und Forschungsprozesse wie auch eine Bedingung dieser Prozesse (vgl. Kron 1999, S. 72 ff.). Eine sozialwissenschaftliche Theorie kann bestimmt werden als ,ein System von allgemeinen Aussagen, das einen systematischen Bezug zu empirisch beobachtbaren sozialen Phänomenen aufweist und diese zu erklären versucht" (Haller 2003, S. 35).

Modelle können eine Vorstufe zur Theoriebildung sein (vgl. Popp 1970) oder sie können Teil einer Theorie sein. Anders aufgefasst ist ein Modell eine formalisierte Theorie (d.h. eine abstraktere Darstellung einer Theorie) (vgl. Mayntz 1967). Sie beziehen sich auf Theorien und gleichzeitig auf Handlungen und können komplexe Zusammenhänge veranschaulichen. Modelle spielen sozusagen eine Vermittlerrolle zwischen Theorien (die sie z. B. für einen Forschungsprozess veranschaulichen können) und der Entwicklung von Konzepten für konkretes pädagogisches Handeln in der Praxis (vgl. Kron 1999, S. 72 ff.). Modelle sind eine Veranschaulichung eines Realitätsausschnitts und bilden gleichzeitig auch Zusammenhänge zwischen Elementen des Realitätsausschnitts ab. Sie vereinfachen und abstrahieren von den je nach Erkenntnisinteresse als jeweils unwichtig gesehenen Merkmalen.

Die Programmplanungsmodelle sind ebenfalls an der Schnittstelle zu verorten: sie sind für Forschung und Praxis mit unterschiedlichen Schwerpunkten relevant und stehen vermittelnd zwischen beiden. Programmplanungshandeln beispielsweise beschrieben und erklärt als Angleichungshandeln (Gieseke 2003) oder als die Ausgestaltung professioneller Antinomien (von Hippel 2011) können als Theorien mittlerer Reichweite bezeichnet werden. Ihre grafischen Abbildungen sind veranschaulichende, abstrahierende Modelle.

Will man Theorien - und hier im engeren Sinne Modelle - miteinander vergleichen, gilt es für einen Theorievergleich, zunächst die Auswahl der zu vergleichenden Theorien zu begründen, d. h. Kategorien der Auswahl, und in einem zweiten Schritt die Vergleichskategorien zu benennen.

\subsection{Programmplanungsmodelle und ihre Differenzierung nach unterschiedlichen Kategorien}

Es gibt verschiedene Programmplanungsmodelle, deren Ähnlichkeiten und Unterschiede sich durch die Einteilung nach verschiedenen Kategorien in einer Systematik beschreiben lassen. Mit den jeweiligen Modellen lassen sich differente Fragestellungen bearbeiten, die Modelle stellen differente Perspektiven auf Programmplanung dar. Bislang gab es wenige Systematiken der Modelle nach unterschiedlichen Kategorien (es wurde hauptsächlich in interaktive und linear-zyklische Modelle unterschieden, vgl. von Hippel und Käpplinger 2017; McLean 2000; oder in den Entstehungshintergrund: klassische theoretische Ansätze, neue, didaktisch aufbereitete, empirisch entwickelte vgl. Gieseke 2000; für Einteilungen der nordamerikanischen Modelle). Eine Systematik ist jedoch wichtig, da sie die unterschiedlichen Anschlüsse und Fragestellungen für Forschung und Praxis aufzeigt und zu einer begründeten Modellauswahl beitragen kann.

Die Modelle sind grundlegend bereichsunabhängig (ob Hauptzweck Weiterbildung oder beigeordnet mit anderen Hauptzwecken als Bildung (vgl. Gieseke 2008) oder allg., beruflich, betrieblich, Volkshochschule etc.), können und werden aber, 
weil sie auch die genügende Abstraktion haben, auf die jeweiligen Bereiche bereichsspezifisch adaptiert. Die Modelle stellen jeweils Teilperspektiven auf Programmplanungsprozesse dar. Oft beinhalten sie auch unterschiedliche Auslegungen von Bildung.

Als Vergleichskategorien ziehe ich die Folgenden heran, da sie besonders anschlussfähig für die Modellauswahl in Forschung und Praxis sind (weitere sind möglich $\left.{ }^{2}\right)$ :

- Aussagekraft zu unterschiedlichen Ebenen: Programmplanung bezieht sich immer auf die Ebene Organisation (makro-/mesodidaktisches Handeln), jedoch weisen die unterschiedlichen Programmplanungsmodelle auch differente Bezüge zu den anderen Ebenen System und Interaktion auf (vgl. zu den Ebenen im Weiterbildungssystem Schrader 2011). Das heißt: Die Modelle lassen sich zusammenfassen nach den Ebenen, zu denen die Programmplanungsmodelle (auch) Aussagen machen. Hieran schließen sich dann jeweils spezifische Fragestellungen an (s. unten).

- (theoretischer) Bezugsrahmen: Als theoretische Bezugspunkte lassen sich - neben anderen - insbesondere pädagogisch-didaktische Theorien, Organisations- und Professionstheorien ausmachen. Sie verweisen auch darauf, welche Fragestellungen besonders gut mit welchen Modellen bearbeitet werden können (z. B. Fragen nach Programmplanung als professionellem Handeln etc.).

- Auffassung von Programmplanung (linear-zyklisch und interaktive Modelle): Linear-zyklische Modelle beschreiben Programmplanung als einen Prozess mit aufeinanderfolgenden idealtypischen Schritten, interaktive Modelle betonen eher den Aushandlungscharakter und die Kontextbezogenheit (vgl. McLean 2000; von Hippel und Käpplinger 2017). Typischerweise enthalten die interaktiven Modelle stärker den/die Planenden und die unterschiedlichen Kontexte als Elemente. Linearzyklische fokussieren eher (aber nicht alle) die Tätigkeitselemente/Aufgaben.

In Tab. 1 habe ich ausgewählte Modelle ${ }^{3}$ (aus dem deutschsprachigen sowie dem englischsprachigen Raum) in den drei Vergleichskategorien verortet. Die Auswahl der deutschsprachigen Modelle ist umfangreich und vollständig, was unterschiedli-

\footnotetext{
2 Als weitere mögliche Kategorien sind z. B. zu nennen: 1. Ziele (beschreiben und/oder erklären): Diese Vergleichskategorie ist jedoch unscharf, da Modelle immer beschreibende und erklärende Elemente enthalten, jedoch mit unterschiedlichen Schwerpunktsetzungen. 2. Teil-/Gesamtmodell: Teilmodelle fokussieren bestimmte Aspekte der Programmplanung, beispielsweise Aushandlungsstrategien oder die Bedarfserhebung. Gesamtmodelle bilden mehr oder weniger den gesamten Prozess der Programmplanung ab. Dieses Kriterium kann individuell für die Modellauswahl herangezogen werden. 3. Grad der Allgemeinheit bzw. Reichweite: Aussagen dazu lassen sich jedoch schwer genau zuordnen, weil die jeweiligen Theorien - hier Modelle - unterschiedliche Fragestellungen mit unterschiedlichen Begriffen, Annahmen, Erklärungsstrategien und Methoden angehen (vgl. Haller 2003, S. 37). Es lässt sich also schwerlich entscheiden, welche Theorie nun besser oder richtig sei (ebd.). Diese Vergleichskategorie ziehe ich nicht heran, da es mir nicht um den Vergleich geht, welches Modell besser oder richtig ist, sondern eher darum, dazu anzuregen, darüber nachzudenken, welches Modell das jeweils passende ist, aber auch welche Aussagen es erlaubt (damit ist eine Form der Reichweite bei den Ebenen angesprochen).

3 Weitere Modelle, die ebenfalls in die obige Systematik eingeordnet werden könnten, sind z. B. von Arnold und Wiegerling (1983) (zirkulär), Robak et al. (2015) (Begründungsmuster, interaktiv), für eine Übersicht über weitere Modelle siehe Gieseke (2000); im englischsprachigen Raum McLean (2000). Darüber hinaus: nicht Modelle, aber Analyseperspektiven auf Programmplanung, z. B. unter neo-institutionalistischen Perspektiven (vgl. von Hippel et al. 2008).
} 
Tab. 1 Nach Kategorien unterschiedene ausgewählte Programmplanungsmodelle (sortiert nach Fokus)

\begin{tabular}{|c|c|c|c|}
\hline \multirow[t]{2}{*}{ Modell } & \multicolumn{3}{|l|}{ Kategorie } \\
\hline & $\begin{array}{l}\text { Programmplanung verstanden } \\
\text { als ... }\end{array}$ & $\begin{array}{l}\text { Bezugsrahmen } \\
\text { der Modelle }\end{array}$ & $\begin{array}{l}\text { Auffassung } \\
\text { von Pro- } \\
\text { grammpla- } \\
\text { nung }\end{array}$ \\
\hline
\end{tabular}

Fokus: übergreifend, Programmplanung als Ebene/Teilbereich der Weiterbildung (, System“)

Mehrebenenmodell der Makro-/mesodidaktische Ebene

WB (Schrader 2011)

Didaktische Handlungsebenen der WB (Flechsig und Haller 1975; Tietgens 1992; Siebert 1982)

Lernkulturen (Gieseke et al. 2009; Fleige und Robak 2016)

Fokus: Mesodidaktische Ebene (,Organisation“)

Suchbewegung (Tietgens 1982)

Angleichungshandeln

(Gieseke 2003)

Gestaltung professioneller Antinomien (von Hippel 2011)

Subjektiv zugewiesene Funktionen betrieblicher WB (von Hippel und Röbel 2016)

Negotiating Power/ Interests (Cervero und Wilson 1994) $)^{\mathrm{a}}$

Planungskulturen (Dollhausen 2008)
Antizipation von Teilnehmendenbedürfnissen/Suchbewegung

Angleichungshandeln

Gestaltung professioneller Antinomien

Zuweisen von subjektiven Funktionen in der beigeordneten Weiterbildung durch Akteure

Aushandeln von Macht und Interessen

Eingebettet in organisationale Kulturen, zwischen pädagogischen und ökonomischen Ansprüchen
Organisations-

Interaktiv

theorien

Didaktische

Theorien

Nicht festge-

legt

Kulturtheorien

Interaktiv

Anthropologische Interaktiv Theorien

Professionsund sozialphi-

losophische

Theorien

Professionstheorien Interaktiv

Soziologische

Theorien

Demokratieund ethische

Theorien

Organisationstheorien

Professionsund sozialphilosophische Theorien

Didaktische

Interactive Model of Program Planning (Caffarella und Daffron 2013) ${ }^{\mathrm{b}}$

Professioneller Handlungszyklus (Weinberg 2000)

Zielgruppenorientierte Programmplanung mit Marketingablaufmodell (Tippelt et al. 2008)
Interaktiver Prozess mit elf Komponenten

Abfolge von (didaktischen) Entscheidungen

Zielgruppenorientierte Planung
Theorien demokratietheorien

Didaktische Theorien

Theorien sozialer Milieus, Marketingtheorien Interaktiv

Linear-zy-

Interaktiv klisch

Linear-Zyklisch 
Tab. 1 Nach Kategorien unterschiedene ausgewählte Programmplanungsmodelle (sortiert nach Fokus) (Fortsetzung)

\begin{tabular}{|c|c|c|c|}
\hline \multirow[t]{2}{*}{ Modell } & \multicolumn{3}{|l|}{ Kategorie } \\
\hline & $\begin{array}{l}\text { Programmplanung verstanden } \\
\text { als ... }\end{array}$ & $\begin{array}{l}\text { Bezugsrahmen } \\
\text { der Modelle }\end{array}$ & $\begin{array}{l}\text { Auffassung } \\
\text { von Pro- } \\
\text { grammpla- } \\
\text { nung }\end{array}$ \\
\hline $\begin{array}{l}\text { Didaktisches Handeln in } \\
\text { der EB (Siebert 2000) }\end{array}$ & $\begin{array}{l}\text { Meso- und Mikrodidaktik (Fokus } \\
\text { Mikro), didaktische Entscheidun- } \\
\text { gen }\end{array}$ & $\begin{array}{l}\text { Didaktische } \\
\text { Theorien }\end{array}$ & Interaktiv \\
\hline $\begin{array}{l}\text { Betriebswirtschaftliche } \\
\text { Weiterbildungsmanage- } \\
\text { mentmodelle }\end{array}$ & Gesamt-/Teilmodelle & $\begin{array}{l}\text { Betriebswirtschaft- } \\
\text { liche Theorien }\end{array}$ & $\begin{array}{l}\text { Linear-zy- } \\
\text { klisch }\end{array}$ \\
\hline $\begin{array}{l}\text { Angebotsentwicklung } \\
\text { (Schlutz 2006) }\end{array}$ & $\begin{array}{l}\text { Als eher mikrodidaktisches Pla- } \\
\text { nen von Bildungsdienstleistungen } \\
\text { mit Dienstleistungsmanagement } \\
\text { und -marketing }\end{array}$ & $\begin{array}{l}\text { Didaktische und } \\
\text { Marketingtheo- } \\
\text { rien }\end{array}$ & $\begin{array}{l}\text { Einzelne } \\
\text { Struktur- } \\
\text { elemente, } \\
\text { eher linear- } \\
\text { zyklisch }\end{array}$ \\
\hline $\begin{array}{l}\text { Question-based approach } \\
\text { to educational design } \\
(\text { Sork 2000) }\end{array}$ & $\begin{array}{l}\text { Stellen von Fragen zu Gestal- } \\
\text { tungselementen }\end{array}$ & $\begin{array}{l}\text { Ethische Theo- } \\
\text { rien }\end{array}$ & Interaktiv \\
\hline
\end{tabular}

${ }^{a}$ Cervero und Wilson (1994) machen deutlich, dass Programmplanung immer im Kontext von Macht- und Interessenskonstellationen stattfindet und es eine besondere Verantwortung für die Erwachsenenbildner bedeutet, diese auszubalancieren und einen demokratischen Planungsprozess zu fördern. Das Modell beinhaltet die Dimensionen der Machtbeziehungen (symmetrisch-asymmetrisch) und der Beziehungen zwischen legitimen Interessen (konsensual-nicht konsensual) mit vier Typen von bestmöglichen Strategien, mit diesen jeweiligen Situationen umzugehen

${ }^{b}$ Das Modell von Caffarella und Daffron (2013) sieht auf den ersten Blick dem Modell der Wissensinseln von Gieseke (2003) ähnlich. Beides sind nicht-lineare Modelle, bei denen es keine Hierarchie der Aufgaben gibt, grafisch als Ellypsen dargestellt, beide um die Jahrtausendwende entstanden (das Modell der Wissensinseln in einer umfangreichen empirischen Studie). Unterschiede liegen u. a. in der empirischen Fundierung und der Anschlussfähigkeit an Professionstheorien (vgl. ausführlich von Hippel und Käpplinger 2017)

${ }^{\mathrm{c}}$ Das Modell von Sork (2000) (question-based approach to educational design) analysiert kritisch, wie der Bedarf mittels Bedarfsanalysen (needs assessments) durch den Einfluss unterschiedlicher Interessensträger konstruiert wird

che Perspektiven betrifft. Die Auswahl der drei englischsprachigen Modelle beruhte auf dem Kriterium, dass es sich exemplarisch um unterschiedliche Perspektiven und um drei besonders in Forschung und Praxis rezipierte Modelle handelt ${ }^{4}$. Leitend für die Auswahl der Modelle (Auswahlkategorien) war mithin die Anwendung der Modelle in Forschung und Praxis (z. B. Arbeit mit den Modellen in Forschungsprojekten und Weiterentwicklung, Zitation in der Literatur etc.).

Die Unterteilung in Tab. 1 und die folgenden Ausführungen können für die Auswahl von Modellen für Forschung und Praxis hilfreich sein. Ein konkreter tiefergehender Vergleich von beispielsweise zwei oder drei Modellen könnte sich an den vorliegenden Beitrag anschließen.

\footnotetext{
${ }^{4}$ Nicht aufgeführt wurden die linear-zyklischen Modelle, die im Prinzip den deutschsprachigen ähnlich sind. Die drei Modelle aus dem englisch-sprachigen Raum werden in den Fußnoten kurz erläutert, da davon ausgegangen wird, dass die deutschsprachigen Modelle eher bekannt sind.
} 
Es gibt eine Fülle von Programmplanungsmodellen (s. oben), die unterschiedliche Fragestellungen erlauben. Das Ziel ist es also nicht, ein neues Modell zu entwickeln, sondern die Modelle zu integrieren und aufzuzeigen, welche Modelle jeweils für bestimmte Fragestellungen in Forschung und Praxis handlungsleitend sein können und den Forschungsgegenstand entsprechend konstruieren.

Im Folgenden sollen die Einordnung der Modelle in die Systematik der Tab. 1 erläutert, die Modelle zusammenfassend beschrieben und mit typischen möglichen Zielen des Modelleinsatzes verknüpft werden.

\subsubsection{Kontexte - Bezüge - Verortung (,System“)}

Hier sind die Modelle zu nennen, die verschiedene Ebenen/Bereiche einschließen (in Tab. 1 der erste Block). Sie sind im engeren Sinne keine spezifischen Programmplanungsmodelle. Sie sind unterschiedlich, erlauben jedoch alle die Analyse von Programmplanung im Bezug und Kontext anderer Bereiche. $\mathrm{Zu}$ nennen sind hier das Mehrebenenmodell (vgl. Schrader 2011), die didaktischen Handlungsebenen (vgl. Siebert 1982, 2000) und die Lernkulturen (Gieseke et al. 2009; Fleige und Robak 2016). Mehrebenenmodell und Lernkulturen beruhen jeweils auf umfangreichen empirischen Studien.

Ziele des Einsatzes dieser Modelle kann es beispielsweise sein, Einflüsse auf Programmplanung zu analysieren, Veränderungen des Weiterbildungssystems abzubilden (Schrader 2011) oder die Gestaltung und Wirkung bildungspolitischer Konzepte auf unterschiedlichen Ebenen zu betrachten (vgl. Gieseke 2008). Die Modelle sind damit insbesondere für die Forschung relevant und für analytische Betrachtungen (in) der Praxis, nicht für die konkrete Gestaltung der Programmplanung.

\subsubsection{Mesodidaktische Ebene („Organisation“)}

In diesem Bereich finden sich Modelle, die das professionelle Handeln der Programmplanenden in verschiedenen Kontexten beschreiben. Als verbindendes Element der meisten der hier genannten Modelle kann man die teilweise widersprüchlichen Erwartungen an und Interdependenzen in der Programmplanung identifizieren (vgl. auch von Hippel 2012).

Hier sind Modelle zu nennen, die den strukturellen Handlungskern des Programmplanungshandelns analysieren: Angleichungshandeln (Gieseke 2000), professionelle Antinomien (von Hippel 2011), Suchbewegung (Tietgens 1982). Des Weiteren sind hier Modelle zu nennen, die Deutungs- und Interpretationszuweisungen fokussieren: Planungskulturen (Dollhausen 2008), subjektive Funktionen (von Hippel und Röbel 2016), Negotiating Power/Interests (Cervero und Wilson 1994). Alle genannten Modelle beruhen auf empirischen Studien (in unterschiedlichem Umfang).

Mit diesen Modellen lassen sich Einflüsse auf die Programmplanung nachzeichnen, gesellschaftliche Auslegungen von Bildung rekonstruieren und das pädagogisch-professionelle Handeln untersuchen. Sie können in der Forschung leitend sein, aber auch die konkrete Reflektion in der Praxis der Programmplanung anregen. 


\subsubsection{Schnittstelle mikrodidaktische Ebene („Interaktion“)}

Die hier zu nennenden Modelle/Modellausschnitte/Perspektiven weisen alle mehr oder weniger eine Schnittstelle zur konkreten Angebotsplanung und zur mikrodidaktischen Planung auf: Wissensinseln (Gieseke 2000), Caffarella und Daffron (2013), alle linear-zyklischen (siehe Tab. 1). Die Modelle beantworten mehr oder weniger umfassend welche (Tätigkeits-)Elemente Programmplanung umfasst. Hier sind vor allem didaktische Theorien grundlegend. Die Zielgruppenorientierte Planung nach einem Marketingablaufmodell stellt mittels der zielgruppenorientierten Planungsmethode der „Produktklinik“ (vgl. von Hippel 2008) als Planungspartizipation (zum Begriff siehe Robak et al. 2013) auch eine Schnittstelle zur Adressatenforschung dar. Entstanden sind die Modelle durch Praxisbeobachtungen oder praxisorientierte Forschungs- und Implementierungsprojekte. Sie eignen sich sowohl für die Forschung für eine genaue Analyse der Programmplanungspraxis als auch für die konkrete Programmplanung als Anleitung.

\section{Ausblick}

Im direkten Anschluss an den vorliegenden Beitrag erscheint es weiterführend sinnvoll zwei oder drei Programmplanungsmodelle direkt vertiefend miteinander zu vergleichen im Sinne eines an Methoden des Theorievergleich (vgl. Haller 2003) angelehnten Modellvergleichs. Vergleichskategorien bzw. -fragen könnten hierzu beispielsweise sein: Entstehungshintergrund, Anwendung in Forschung und Praxis, Komplementarität/Überschneidungen/Inkompatibilitäten, Erklärungsanspruch und -fähigkeit bzgl. bestimmter Ziele und Fragestellungen, Potenzial zur weiteren Theoriebildung und/oder Praxisveränderung. Auch ein empirischer Theorienvergleich ist vorstellbar. Ziele der Modellvergleiche könnten in der Weiterentwicklung und auch u. U. Integration und Kombination der Modelle liegen.

Als Forschungs- und Entwicklungsdesiderate in Bezug auf Programmplanungsmodelle lassen sich aus den oben genannten Ausführungen folgende Punkte weiterführen. Ein Desiderat stellt die vertiefte Verknüpfung der Programmplanungsmodelle mit der Adressat/inn/en- und Teilnehmer/innenforschung dar. Hier könnte beispielsweise gefragt werden, wie Programmplanungsmodelle Schnittstellen zu den Adressaten- und Teilnehmenden beinhalten um dann verknüpft mit Programmforschung Rezeptionsstudien durchzuführen (s. oben). Ein weiteres Forschungsdesiderat ist zu sehen in verknüpften empirischen Analysen von Planungsprozessen und den Programmen. Aus solchen Analysen könnten wiederum die bestehenden Programmplanungsmodelle erweitert werden um diese Verknüpfung mit den Handlungsergebnissen, den Programmen (die oft nur indirekt in den Modellen abgebildet sind), beispielsweise im Sinne von Mustern und Typen (Programmplanungskulturen). Auch die systematische bereichsspezifische Adaptierung einzelner Modelle stellt ein Forschungs- und Entwicklungsdesiderat dar. Für die Professionalisierung ist es wiederum wichtig, die unterschiedlichen Modelle in Studiengängen und Fortbildungen für Erwachsenenbildner zu bearbeiten. 
Open Access Dieser Artikel wird unter der Creative Commons Namensnennung 4.0 International Lizenz (http://creativecommons.org/licenses/by/4.0/deed.de) veröffentlicht, welche die Nutzung, Vervielfältigung, Bearbeitung, Verbreitung und Wiedergabe in jeglichem Medium und Format erlaubt, sofern Sie den/die ursprünglichen Autor(en) und die Quelle ordnungsgemäß nennen, einen Link zur Creative Commons Lizenz beifügen und angeben, ob Änderungen vorgenommen wurden.

\section{Literatur}

Arnold, R., \& Wiegerling, H.-J. (1983). Programmplanung in der Weiterbildung. Bedarfsorientierung, Ausgewählte Planungsstrategien, Institutionelle Einflüsse. Frankfurt am Main: Diesterweg.

Caffarella, R., \& Daffron, S. (2013). Planning programs for adult learners. A practical guide. San Francisco: Jossey-Bass.

Cervero, R. M., \& Wilson, A.L. (1994). Planning responsibly for adult education. A guide to negotiating power and interests. San Francisco: Jossey-Bass.

DIE (2017). Bibliographie Programmanalysen. https://www.die-bonn.de/institut/dienstleistungen/service stellen/programmforschung/bibliographie/analyse.aspx. Zugegriffen: 10.08.2017.

Dollhausen, K. (2008). Planungskulturen in der Weiterbildung. Angebotsplanungen zwischen wirtschaftlichen Erfordernissen und pädagogischem Anspruch. Bielefeld: wbv.

Flechsig, K.-H., \& Haller, H.-D. (1975). Einführung in didaktisches Handeln. Stuttgart: Klett.

Fleige, M., \& Robak, S. (2016). Lehr-Lernkulturen in der Erwachsenenbildung. In R. Tippelt \& A. von Hippel (Hrsg.), Handbuch Erwachsenenbildung/Weiterbildung 6. Aufl. Wiesbaden: Springer. https:// doi.org/10.1007/978-3-531-20001-9_35-1.

Gieseke, W. (Hrsg.). (2000). Programmplanung als Bildungsmanagement? Qualitative Studie in Perspektivverschränkung. Begleituntersuchung des Modellversuchs, Entwicklung und Erprobung eines Berufseinführungskonzepts für hauptberufliche Erwachsenenbilder/innen'. Recklinghausen: Bitter.

Gieseke, W. (2003). Programmplanungshandeln als Angleichungshandeln: die realisierte Vernetzung in der Abstimmung von Angebot und Nachfrage. In W. Gieseke (Hrsg.), Institutionelle Innensichten der Weiterbildung (S. 189-211). Bielefeld: wbv.

Gieseke, W. (2008). Bedarfsorientierte Angebotsplanung in der Erwachsenenbildung. Bielefeld: wbv.

Gieseke, W., Robak, S., \& Wu, M.-L. (Hrsg.). (2009). Transkulturelle Perspektiven auf Kulturen des Lernens. Bielefeld: transcript.

Gieseke, W. (2015). Professionalität und Professionalisierung in der Erwachsenenbildung/Weiterbildung. In R. Tippelt \& A. von Hippel (Hrsg.), Handbuch Erwachsenenbildung/Weiterbildung 6. Aufl. Wiesbaden: Springer VS. https://doi.org/10.1007/978-3-531-20001-9_52-1.

Gnahs, D. (1995). Analysen von Veranstaltungsverzeichnissen und -ankündigungen auf Informationsvielfalt und -genauigkeit. In R. von Bardeleben, D. Gnahs, E. M. Krekel \& B. Seusing (Hrsg.), Weiterbildungsqualität - Konzepte, Instrumente und Kriterien (S. 29-43). Bielefeld: wbv.

Haller, M. (2003). Soziologische Theorie im systematisch-kritischen Vergleich. Wiesbaden: VS.

Herre, P. (2015). Recherche-Projekt Programmforschung Sammlungs- und Archivierungspraxis von Programmen in europäischen Ländern. DIE-Projektbericht. http://www.die-bonn.de/docs/Herre_2015_ DIE-Projektbericht-Europaeische\%20Recherche.pdf. Zugegriffen: 10.08.2017.

Heuer, U., \& Robak, S. (2000). Programmstrukturen in konfessioneller Trägerschaft - exemplarische Programmanalysen. In W. Gieseke (Hrsg.), Programmplanung als Bildungsmanagement? Qualitative Studie in Perspektivverschränkung (S. 115-209). Recklinghausen: Bitter.

von Hippel, A. (2008). Die Produktklinik - eine Methode zur nachfrageorientierten Planung von Angeboten wissenschaftlicher Weiterbildung. Report-Zeitschrift für Weiterbildungsforschung, 31(1), 42-51. https://doi.org/10.3278/rep0801w042.

von Hippel, A. (2011). Programmplanungshandeln im Spannungsfeld heterogener Erwartungen: ein Ansatz zur Differenzierung von Widerspruchskonstellationen und professionellen Antinomien. ReportZeitschrift für Weiterbildungsforschung, 34(1), 45-57.

von Hippel, A. (2012). Programmplanung als professionelles Handeln - „Angleichungshandeln“ und „Aneignungsmodi“ im aktuellen Diskurs der Programm- und Professionsforschung. In B. Käpplinger, S. Robak \& S. Schmidt-Lauff (Hrsg.), Engagement für die Erwachsenenbildung - Ethische Bezugnahmen und demokratische Verantwortung. Festschrift für Wiltrud Gieseke (S. 131-143). Wiesbaden: Springer VS.

von Hippel, A., \& Röbel, T. (2016). Funktionen als akteursabhängige Zuschreibungen in der Programmplanung betrieblicher Weiterbildung. Report - Zeitschrift für Weiterbildungsforschung, 39(1), 61-81. https://doi.org/10.1007/s40955-016-0053-1. 
von Hippel, A., \& Käpplinger, B. (2017). Models of program planning in different countries. In B. Käpplinger, S. Robak, M. Fleige, A. von Hippel \& W. Gieseke (Hrsg.), Cultures of program planning in adult education: concepts, research results and archives Studies in pedagogy, andragogy, and gerontagogy. Frankfurt am Main: Peter Lang.

von Hippel, A., Fuchs, S., \& Tippelt, R. (2008). Weiterbildungsorganisationen und Nachfrageorientierung - neo-institutionalistische Perspektiven. Zeitschrift für Pädagogik, 54(5), 663-678.

Jarvis, P. (2006). Adult education \& lifelong learning. Theory and practice. Abingdon: Routledge.

Käpplinger, B. (2008). Programmanalysen und ihre Bedeutung für pädagogische Forschung. Forum Qualitative Sozialforschung, 9(1), Art. 37. http://www.qualitative-research.net/index.php/fqs/article/view/ 333.

Käpplinger, B., Robak, S., Fleige, M., von Hippel, A., \& Gieseke, W. (Hrsg.). (2017). Cultures of program planning in adult education: concepts, research results and archives. Studies in pedagogy, andragogy, and gerontagogy. Frankfurt am Main: Peter Lang.

Kron, F. W. (1999). Wissenschaftstheorie für Pädagogen. München: Reinhardt. Mit 9 Tabellen

Mayntz, R. (1967). Modellkonstruktion: Ansatz, Typen und Zweck. In R. Mayntz (Hrsg.), Formalisierte Modelle in der Soziologie. Neuwied: Luchterhand.

McLean, S. (2000). "Between rationality and politics": autobiographical portraits of adult education programme planning. International Journal of Lifelong Education, 19(6), 493-505.

Nolda, S. (2015). Programmanalyse in der Erwachsenenbildung/Weiterbildung - Methoden und Forschungen. In R. Tippelt \& A. von Hippel (Hrsg.), Handbuch Erwachsenenbildung/Weiterbildung 6. Aufl. Wiesbaden: Springer VS. https://doi.org/10.1007/978-3-531-20001-9_22-1.

Popp, W. (1970). Die Funktion von Modellen in der didaktischen Theorie. In G. Dohmen (Hrsg.), Unterrichtsforschung und didaktische Theorie (S. 49-60). München: Piper.

Robak, S. (2012). Programmanalysen: Einführung in die Erstellung von Codesystemen. http://www.diebonn.de/institut/dienstleistungen/servicestellen/programmforschung/methodische_handreichungen/ codiersysteme/Programmanalyse-Codesysteme-Robak.pdf. Zugegriffen: 10.08.2017.

Robak, S., Pohlmann, C., \& Heidemann, L. (2013). Zur Analyse von Folgewirkungen bildungspolitischer Strukturentscheidungen auf Bildungs- und Planungspartizipation am Beispiel von Bildungsurlaub. Wiesbaden: Springer.

Robak, S., Rippien, H., Heidemann, L., \& Pohlmann, C. (2015). Bildungsurlaub - Planung, Programm und Partizipation. Eine Studie in Perspektivverschränkung. Frankfurt am Main: Peter Lang.

Schlutz, E. (2006). Bildungsdienstleistungen und Angebotsentwicklung. Münster: Waxmann.

Schrader, J. (2011). Struktur und Wandel der Weiterbildung. Bielefeld: wbv.

Schrader, J., \& Ioannidou, A. (2011). Ziele, Inhalte und Strukturen der Erwachsenenbildung im Spiegel von Programmanalysen. In T. Fuhr, P. Gonon \& C. Hof (Hrsg.), Handbuch der Erziehungswissenschaft. 4. Erwachsenenbildung - Weiterbildung (S. 259-269). Paderborn: Schöningh.

Siebert, H. (1982). Programmplanung als didaktisches Handeln. In E. Nuissl (Hrsg.), Taschenbuch der Erwachsenenbildung (S. 100-121). Baltmannsweiler: Burgbuecherei Schneider.

Siebert, H. (2000). Didaktisches Handeln in der Erwachsenenbildung. Didaktik aus konstruktivistischer Sicht. Neuwied: Luchterhand.

Sork, T. J. (2000). Planning educational programs. In A. Wilson \& E. R. Hayes (Hrsg.), Handbook of adult and continuing education (S. 171-190). San Francisco: Jossey-Bass.

Tietgens, H. (1982). Angebotsplanung. In E. Nuissl (Hrsg.), Taschenbuch der Erwachsenenbildung (S. 122-144). Baltmannsweiler: Burgbuecherei Schneider.

Tietgens, H. (1992). Reflexionen zur Erwachsenendidaktik. Bad Heilbrunn: Klinkhardt.

Tippelt, R., Reich, J., von Hippel, A., Barz, H., \& Baum, D. (2008). Milieumarketing implementieren. Weiterbildung und soziale Milieus, Bd. 3. Bielefeld: wbv.

Weinberg, J. (2000). Einfuihrung in das Studium der Erwachsenenbildung. Bad Heilbrunn: Klinkhardt. 